\title{
Resonant RF Cavity Based Beam Chopping for Precise Control over Electron Arrival Time Distribution
}

\author{
Erik Kieft $^{1 *}$, Jasper van Rens ${ }^{2}$, Wouter Verhoeven ${ }^{2}$, Peter Mutsaers ${ }^{2}$ and Jom Luiten ${ }^{2}$ \\ 1. Thermo Fisher Scientific, Eindhoven, the Netherlands. \\ 2. Department of Applied Physics, Coherence and Quantum Technology Group, Eindhoven University of \\ Technology, Eindhoven, the Netherlands. \\ * Corresponding author: erik.kieft@thermofisher.com
}

Modulation (i.e., pulsing) of electron beams has been used in electron microscopy, mostly in a pumpprobe configuration, for achieving time resolution well beyond the typical framerates of cameras and other detectors [e.g., 1,2]. Meanwhile, there has been lively debate on the possible role of dose rate beside total dose as a factor governing reversible as well as irreversible loss of contrast in sensitive samples under investigation in high-resolution (S)TEM [3,4]. By extension, also the arrival time distribution of electrons in the beam may have a similar influence, leading to possible new application of time-modulated beams in the study of dose sensitive specimens $[5,6]$.

Beside laser-pulsed photoemission as routinely applied in Ultrafast EM, fast deflection of an otherwise conventional, high-coherence electron beam is a potential candidate for achieving beam modulation, in particular for high pulse rates. Here, we discuss several novel aspects of a resonant beam blanker at RF frequencies that allow its efficient and relatively straightforward implementation into state-of-the art transmission electron microscopes, while preserving quality of the beam, maintaining compatibility with other beam manipulation techniques, and at the same time being able to access sub-ps timescales.

A first realization has been that partially filling a resonant RF cavity with a high-permittivity, low-loss dielectric material reduces both the physical dimensions and power consumption of that cavity, for a given resonance frequency and on-axis deflection field. A recent step in optimization of that geometry was to move out the outer radial wall of the cavity while keeping most of the mode energy concentrated towards the axis, thereby reducing losses due to wall dissipation [7]. A second adaptation is to simply adjust the microscope optics such that a crossover of the beam is at the (effective) deflection pivot point of the cavity. Admittedly, in the present implementation this goes at the cost of a degree of freedom in the optics of electron probe formation, but this is not a fundamental limitation of the technique. It is this configuration in which the setup has recently been used in diffraction mode to study the structural decay of an industrially highly relevant, but challenging material from an imaging and characterization point of view $-\mathrm{MgCl}_{2}$ as a Ziegler-Natta catalyst in the high-volume production of polyethylene $[5,6]$.

A subsequent third step has been the implementation of a so-called dual mode version of the cavity, where the degeneracy of the $\mathrm{TM}_{110}$ deflecting mode is deliberately broken, and the two modes are separated by (in this case) a $75 \mathrm{MHz}$ difference frequency. When both modes are energized to a significant extent, the electron beam scribes a Lissajous pattern in the plane of the blanking aperture, such that with a properly selected aperture size and position, pulses at the difference frequency can be generated [8]. Thereby, extra flexibility is introduced in terms of temporal pulse separation, and also the pulse repetition rate is brought into a range that is accessible for further manipulation or analysis using other, more conventional techniques. A well-established signal-mixing technique is used for phase-locking the resulting electron pulses to the arrival times of pulses from a fs laser oscillator [9], to within $100 \mathrm{fs}$. Alternatively, the cavity 
can be operated in a free-running way (for use without a laser oscillator), but still having both deflection modes phase locked to one another for overall phase stability.

After realization of this dual-mode pulse generation, our efforts have been focused on understanding and eliminating any remaining instabilities in the system (including laser pointing, and mode stability in the cavity), thereby enabling unsupervised operation of the microscope on the typical timescales often required for high-quality time-resolved experiments, of an hour or more. One finding was a gradual, unbalanced electrical charging of the inner surface of the dielectric cylinder leading to beam drift on very long timescales, an effect that was anticipated but previously not observed during shorter acquisitions. The effect may be mitigated by applying a thin conductive layer on the inner bore's surface, to drain the electrical charges slowly building up on the material, while leaving the resonant modes sufficiently undisturbed [10].

\section{References:}

[1] AH Zewail, Annu.Rev.Phys.Chem. 57 (2006) p. 65.

[2] T LaGrange et al., Micron 43 (2012) p 1108.

[3] C Kisielowski et al., Phys. Rev. B 88 (2013), 024305.

[4] J Barthel, M Lentzen and A Thrust, Ultramicroscopy 176 (2017) pp 37-45.

[5] C Kisielowski et al., Adv. Funct. Mater. (2019), 1807818.

[6] C Kisielowski et al., these proceedings.

[7] W Verhoeven et al., https://arxiv.org/abs/1811.02243

[8] JFM van Rens et al., Appl. Phys. Lett. 113 (2018), 163104.

[9] FB Kiewiet et al, Nucl.Instrum.Meth.Phys.Res.A 484 (2002), 619.

[10] This work is part of an Industrial Partnership Programme of the Netherlands Organisation for Scientific Research (NWO). The authors would like to thank E.H. Rietman, I. Koole, H.A. van Doorn, and A.H. Kemper for their invaluable technical support.
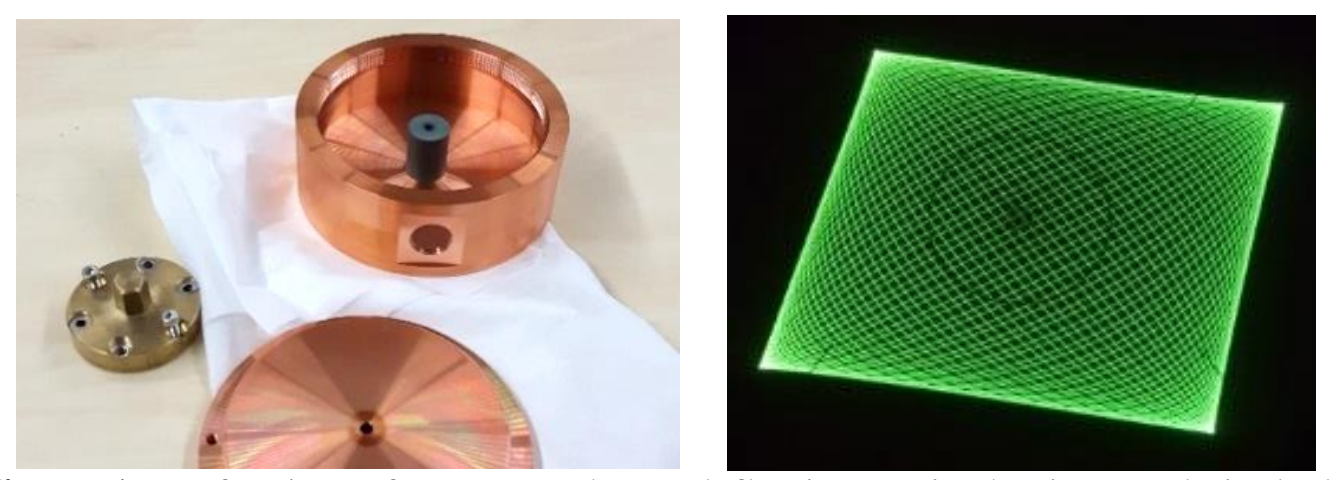

Figure 1. Left: view of an (opened) RF deflection cavity having a relatively large outer diameter for energy-efficient operation; in this case a single-mode version. Right: Lissajous pattern imaged on the fluscreen of the microscope. Clear separation of the lines of the pattern demonstrates good phase stability between the modes. 